\title{
Influência da restrição de água e ração durante a fase pré-inicial no desempenho de frangos de corte até os 42 dias de idade
}

\author{
Lisiane Fernandes Soares ${ }^{1}$, Andréa Machado Leal Ribeiro², Antônio Mário Penz Júnior², \\ André Ghiotti ${ }^{3}$
}

\footnotetext{
${ }^{1}$ Departamento de Bioquímica e Microbiologia da UTFPR, Campus Dois Vizinhos, Estrada da Boa Esperança, Km 04, s/n Dois Vizinhos - PR 2 Departamento de Zootecnia - Universidade Federal do Rio Grande do Sul, Porto Alegre - RS.

${ }^{3}$ Bolsista de Iniciação Científica - Departamento de Zootecnia - Universidade Federal do Rio Grande do Sul, Porto Alegre - RS.
}

RESUMO - Dois experimentos foram conduzidos para avaliar a influência da restrição de água e ração durante a fase préinicial no desempenho de frangos de corte e no desenvolvimento dos órgãos (coração, fígado, moela e intestinos). Em cada experimento utilizaram-se 480 pintos machos, Ross, distribuídos em cinco níveis (0, 10, 20, 30 e 40\%) de restrição de água ou de ração durante os primeiros sete dias de idade. As aves foram alimentadas com a mesma dieta, em composição nutricional, em todos os tratamentos. Com restrição de água ou de ração, foram observadas reduções lineares no desempenho nos primeiros sete dias de idade e redução absoluta do peso dos órgãos, no entanto, o peso relativo não foi alterado, com exceção da moela, cujo peso relativo aumentou com a restrição. Após os 7 dias de idade, verificou-se crescimento compensatório dos frangos, sobretudo na semana subseqüente à restrição (8 a 14 dias). Aos 42 dias, as aves apresentaram peso corporal semelhante, independentemente do tipo de restrição. Nas aves sob restrição de água, a conversão alimentar no período de 8 a 14 dias de idade foi melhor nas aves submetidas a restrição prévia, enquanto, entre aquelas sob restrição de ração, essa diferença não ocorreu. O percentual de hematócrito sanguíneo aumentou linearmente aos 7 dias de idade com o aumento da restrição hídrica e aos 3 dias idade, com o aumento da restrição de ração. Em ambos os experimentos, não houve influência das restrições na uniformidade do lote e no rendimento de carcaça e dos cortes. O consumo de água foi mais independente que o consumo de ração, pois apresentou menor redução quando as aves estavam sob restrição de ração.

Palavras-chave: aves, crescimento compensatório, matéria seca

\section{Effect of water and feed restriction during pre-starter phase on the performance of broiler up to 42 days of age}

\begin{abstract}
Two experiments were conducted to evaluate the influence of water and feed restriction during the prestarter phase on the broilers performance and organs development (heart, liver, gizzard and intestines). In each experiment, a 480 males Ross chicks was allotted to a five levels $(0,10,20,30$ and 40\%) of water or feed restriction during the first seven days of age. The broilers were fed with the same diet, in nutritional composition, in all treatments. With water or feed restriction, it was observed a linear reduction in the performance during de first seven days of age and reduction in absolute organs weight, however the relative weight (RW) was not affected, except for gizzard that increased with restriction. After 7 days of age, it was observed a compensatory gain of the broilers, over all in the subsequent week ( 8 to 14 days). At 42 days, the birds showed similar body weight independently of the type of restriction. In the birds under water restriction, the feed conversion in the period from 8 the 14 days of age was better birds submitted to previous restriction, while, among those under ration restriction, this difference did not occur. Percentage of blood hematocrit linearly increased at 7 days of age with the increase of water restriction, and at three days of age with feed restriction. In both experiments there was no influence of the restrictions in the lot uniformity and in carcass and parts yield. The water intake was more independent than feed intake, therefore showed lower reduction when the birds were under feed restriction.
\end{abstract}

Key Words: birds, compensatory growth, dry matter

\section{Introdução}

Na produção avícola, a deficiência no fornecimento de água pode ocasionar crescimento retardado, falta de uniformidade e problemas de saúde em frangos de corte
(Counotte, 2003). A água contribui em diversos processos vitais, como digestão (hidrólise de proteínas, gorduras e carboidratos), absorção e circulação de nutrientes para respiração, temperatura corporal e funcionamento do sistema nervoso. Atua ainda no transporte de hormônios, na 
lubrificação das articulações e na visão e audição (Lloyd et al., 1978; Nilipour \& Butcher, 1998).

O desenvolvimento do trato gastrintestinal durante os primeiros estádios pós-eclosão é muito importante. Segundo Dibner (1996), frangos de corte passam por uma grande modificação ao eclodirem e têm pouco tempo de adaptação desde o início da vida pós-embrionária para expressar todo o seu potencial genético. Entre os fatores que podem interferir no crescimento inicial desses animais, destacam-se a qualidade e a quantidade de água e de alimento disponível. Nitsan et al. (1991) relataram que o peso dos órgãos responsáveis pela digestão em frangos de corte aumenta de forma linear durante os primeiros 15 dias após o nascimento. $\mathrm{O}$ adequado fornecimento de alimentos e água na primeira semana de vida torna-se, portanto, essencial para o bom desempenho dos frangos ao longo do período de criação.

Aves sem acesso à água, até mesmo por poucas horas, especialmente em ambientes quentes, interrompem o crescimento e podem apresentar maior susceptibilidade a doenças (Nilipour \& Butcher, 1998). Sabe-se que restrição de água propicia a redução no consumo de alimento (Brooks, 1994; Larbier \& Leclercq, 1994). Quando a água é oferecida à vontade, as aves desenvolvem um padrão bem característico de ingestão de alimento. Qualquer fator que interfere na ingestão de água altera a ingestão de alimento e vice-versa (Macari, 1995).

A restrição é sempre vista como uma influência negativa no crescimento dos frangos. No entanto, as restrições alimentares, em curto espaço de tempo e em uma fase que permita a recuperação dos frangos, têm resultado em um fenômeno chamado crescimento acelerado, que contribui para melhores resultados econômicos na criação. Esse crescimento acelerado está relacionado, provavelmente, à redução nas exigências de energia de manutenção das aves durante a restrição alimentar (Plavnik \& Hurvitz, 1985). Ainda assim, verifica-se inconsistência nos resultados de várias pesquisas, principalmente quanto ao consumo de ração e à existência de crescimento acelerado. Do mesmo modo, em virtude da intensa relação do consumo de água com consumo de alimento, a restrição de água também poderia resultar em fenômenos posteriores de crescimento acelerado.

Este trabalho foi realizado com o objetivo de avaliar a importância do fornecimento de água e de ração nos primeiros sete dias de idade de frangos de corte e a influência da restrição de água ou de ração no desempenho, no crescimento compensatório, no teor de matéria seca dos tecidos, no hematócrito e no rendimento de carcaça dessas aves no período pós-restrição de água ou de ração até os 42 dias de idade.

\section{Material e Métodos}

Dois experimentos foram realizados no Laboratório de Ensino Zootécnico da UFRGS no ano de 2004. Em cada experimento foram utilizados 420 pintos machos, de 1 dia de idade, da linhagem Ross 308. As aves foram criadas em salas climatizadas, em quatro baterias, cada uma com dez gaiolas de $0,73 \mathrm{~m}^{2}$, equipadas com dois bebedouros do tipo infantil e um comedouro tipo calha. Aos 21 dias de idade, os animais foram transferidos para gaiolas de $0,84 \mathrm{~m}^{2}$, providas de comedouros e bebedouros do tipo calha.

Os tratamentos no experimento 1 foram constituídos de níveis crescentes $(0,10,20,30$ e $40 \%)$ de restrição de água no período de 1 a 7 dias de idade e no experimento 2 , de níveis crescentes de restrição de ração no mesmo período. Os consumos de água e de ração na primeira semana foram estimados em um lote de seis repetições, 14 aves/repetição, com peso inicial similar ao das aves experimentais, alojado no mesmo local com três dias de antecedência ao período experimental. Os valores de consumo de água ou de ração deste lote (à vontade) foram usados como referência para constituir as restrições impostas às aves experimentais.

Foi utilizada a mesma dieta comercial em todos os tratamentos, conforme a fase de criação. A dieta pré-inicial forneceu $3.100 \mathrm{kcal} \mathrm{EM} / \mathrm{kg}, 21 \% \mathrm{~PB}, 1 \% \mathrm{Ca}, 0,5 \% \mathrm{P}$ disponível, $1,26 \%$ Lis total e $0,94 \%$ de Met+Cis total. As dietas inicial e de crescimento, respectivamente, tiveram $3.180 \mathrm{kcal}$ $\mathrm{EM} / \mathrm{kg}, 20 \% \mathrm{~PB}, 1 \% \mathrm{Ca}, 0,45$ P disponível, 1,17\% Lis total, $0,9 \% \mathrm{Met}+\mathrm{C}$ is total, $3.200 \mathrm{kcal} \mathrm{EM} / \mathrm{kg}, 19,5 \% \mathrm{~PB}, 0,95 \% \mathrm{Ca}$, $0,45 \% \mathrm{P}$ disponível, $1,03 \%$ Lis total e $0,82 \%$ Met + Cis. A água fornecida aos animais foi considerada potável pelo Laboratório de Análise de solos da UFRGS.

Água e ração foram fornecidas uma vez ao dia, para todos os animais, sempre no mesmo horário e foram medidas o consumo e a quantidade fornecida diariamente até os 7 dias de idade. No experimento de restrição de água, foi medida a perda de água por evaporação dos bebedouros para correção na quantidade consumida. Após os 7 dias de idade, o consumo de ração foi calculado semanalmente e não houve mais medição de consumo de água. Os frangos foram pesados semanalmente até os 42 dias de idade. Semanalmente, uma ave de peso médio por repetição foi sacrificada para determinação do peso corporal e do teor de matéria seca dos órgãos coração, proventrículo, moela, intestinos delgado e grosso e fígado+vesícula. As pesagens dos órgãos foram feitas imediatamente após o abate. Os intestinos delgado e grosso, o proventrículo e a moela tiveram seus conteúdos esvaziados antes da pesagem. A partir dos pesos absolutos, foram calculados os pesos de 
órgãos relativos, utilizando-se como denominador o peso vivo da ave.

Para estudar o percentual de hematócrito, o sangue foi coletado de uma veia da asa de uma ave de cada repetição (6 aves/tratamento), diretamente em um microcapilar aos 3 , $7,14,21,28,35$ e 42 dias de idade. Ao completar 42 dias de idade, as aves foram abatidas após jejum de 8 horas; foram pesadas individualmente, sangradas por dois ou três minutos, escaldadas a uma temperatura de $60^{\circ} \mathrm{C}$, depenadas com depenadeira elétrica semi-automática e evisceradas manualmente. As carcaças foram resfriadas em água com gelo durante aproximadamente 40 minutos, simulando um chiller (resfriamento realizado na indústria), e em seguida foram pesadas para obtenção do peso de carcaça resfriada, sem gordura abdominal, sem pés e sem cabeça. Depois, foram separados e pesados individualmente os cortes peito, dorso, coxas, pernas e asas de cada ave. O rendimento de carcaça e dos cortes foi obtido dividindo-se o peso das partes pelo peso da carcaça.

O delineamento experimental utilizado foi o inteiramente casualisado (DIC), com cinco tratamentos, cada um com seis repetições. A análise de variância, a comparação de médias pelo teste Tukey e a análise de regressão foram feitas utilizando o módulo GLM do programa Statistical Analysis System (SAS, 2001).

\section{Resultados e Discussão}

No experimento de restrição de água, a restrição foi efetivamente imposta de acordo com os níveis propostos. $\mathrm{O}$ consumo de ração foi afetado linear e negativamente, confirmando a relação direta entre essas duas variáveis. Com a restrição, houve também decréscimo linear no peso corporal e no ganho de peso (GP) (Tabela 1). Viola (2003), em experimento para avaliação dos mesmos níveis de restrição de água, porém até os 21 dias de idade, também observou redução linear no consumo de alimento. A conversão alimentar (CA), no entanto, não foi afetada pela restrição de água na primeira semana, o que difere do resultado obtido por Viola (2003), que observou, para o mesmo período, que a CA piorou à medida que os níveis de restrição aumentaram.

No período de 8 a 14 dias, imediatamente após a restrição de água, foi observado maior consumo de água entre as aves submetidas a restrição prévia (Tabela 2). Esse aumento foi proporcional ao nível de restrição anterior, conforme indica a regressão linear. $O$ consumo de ração continuou afetado pela restrição de água da semana anterior. No entanto, quando calculado proporcionalmente ao peso metabólico, foi maior no grupo com restrição de $40 \%$ $\left(1,60 \mathrm{~kg} / \mathrm{kg}^{0,75}\right)$ em comparação àquele sem restrição $\left(1,30 \mathrm{~kg} / \mathrm{kg}^{0,75}\right)$. Esse é um comportamento típico de animais em crescimento compensatório. Leu et al. (2002) observaram que aves sob restrição de ração quando alimentadas à vontade podem apresentar inicialmente maior consumo inicial de ração (hiperfagia) em virtude do estado fisiológico que se encontram. Apesar de o ganho de peso nesta fase não ter apontado relação de regressão clara com a retrição de água do período anterior, os dados comprovam que o grupo com $40 \%$ de restrição, que na primeira semana apresentou ganho de peso $41 \%$ inferior ao do grupo controle, alcançou aos 14 dias 7,4\% a mais de ganho de peso.

A conversão alimentar melhorou de forma linear nas aves com maior restrição de água no período anterior. $\mathrm{O}$ peso corporal continuou linear e negativamente reduzido

Tabela 1 - Consumos de água (CA) e de ração $(C R)$, relação $C A: C R$, peso corporal (PC), ganho de peso (GP) e conversão alimentar (CA) de frangos de corte submetidos a diferentes níveis de restrição de água durante os primeiros sete dias de idade

Table 1 - Water intake (WI), feed intake (FI), WI:FI ratio, body weight (BW), weight gain (WG) and feed conversion (FC) of broilers submitted to different levels of water restriction during the first seven days of age

\begin{tabular}{|c|c|c|c|c|c|c|}
\hline $\begin{array}{l}\text { Nível de } \\
\text { restrição (\%) } \\
\text { Restriction level }\end{array}$ & $\begin{array}{l}\text { Consumo de } \\
\text { água }(\mathrm{mL})^{*} \\
\text { Water intake }\end{array}$ & $\begin{array}{l}\text { Consumo de } \\
\text { ração }(\mathrm{g}) * \\
\text { Feed intake }\end{array}$ & $\begin{array}{c}\text { Relação CA:CR } \\
\text { WI:FI ratio }\end{array}$ & $\begin{array}{c}\text { Peso corporal }(\mathrm{g})^{*} \\
\text { Body weight }\end{array}$ & $\begin{array}{l}\text { Ganho de } \\
\text { peso }(\mathrm{g})^{*} \\
\text { Weight gain }\end{array}$ & $\begin{array}{c}\text { Conversão alimentar }(\mathrm{g} / \mathrm{g}) \\
\text { Feed conversion }\end{array}$ \\
\hline 0 & $443 \mathrm{a}$ & $148 \mathrm{a}$ & 2,99 & $184 \mathrm{a}$ & $141 \mathrm{a}$ & 1,11 \\
\hline 10 & $384 b$ & $128 b$ & 3,00 & $161 \mathrm{ab}$ & $118 \mathrm{ab}$ & 1,09 \\
\hline 20 & $342 c$ & $111 \mathrm{c}$ & 3,08 & $140 b c$ & $98 b c$ & 1,14 \\
\hline 30 & $299 d$ & $110 \mathrm{c}$ & 2,72 & $135 b c$ & $92 \mathrm{bc}$ & 1,20 \\
\hline 40 & $261 \mathrm{e}$ & $101 \mathrm{c}$ & 2,58 & $125 \mathrm{c}$ & $82 c$ & 1,23 \\
\hline $\mathrm{CV} \%$ & 2,2 & 5,6 & & 10,8 & 15,1 & 14,0 \\
\hline Valor de P & 0,0001 & 0,0001 & & 0,0001 & 0,0001 & 0,58 \\
\hline
\end{tabular}

$P$ value

$\mathrm{PC}=186,2-1,73 \mathrm{x} ; \mathrm{R}^{2}=0,79 ; \mathrm{P}<0,0001$ 
pela severidade da restrição. O crescimento compensatório tem a vantagem de modificar o padrão de crescimento das aves, principalmente porque no período de restrição o peso corporal dos animais é reduzido, diminuindo também a exigência de nutrientes de mantença. Na fase de realimentação, essa modificação resulta em maior eficiência de uso dos nutrientes para o crescimento (Doyle \& Leeson, 1997), o que explica os melhores índices de conversão alimentar.

$\mathrm{Na}$ análise do período de 1 a 21 dias (Tabela 2), não houve diferença entre os níveis de restrição de água para o consumo de água, o ganho de peso e o peso corporal. $\mathrm{O}$ consumo de ração (CR), no entanto, permaneceu negativamente relacionado à restrição da primeira semana e a conversão alimentar continuou positivamente influenciada pela RAg, como verificado na semana imediatamente posterior à restrição. Apesar de não-significativa, houve uma diferença de mais de $40 \mathrm{~g}$ no peso corporal entre as aves submetidas a restrição de $40 \%$ e aquelas controle (sem restrição), o que sugere serem necessários mais que 14 dias para a recuperação do peso corporal padrão.

No período de 22 a 42 dias (Tabela 2), a restrição prévia de água não influenciou o consumo de ração, o ganho de peso ou o peso corporal. No entanto, a conversão alimentar nos frangos submetidos a restrição de água continuou melhorando de forma linear, conforme a magnitude da restrição. Esse resultado evidencia a ocorrência de crescimento compensatório, como observado por Lawrence \& Fowler (2002) e Fontana et al. (1993).

Do mesmo modo que não houve diferenças no peso corporal aos 42 dias, também não houve diferença no

Tabela 2 - Consumos de água (CAg) e de ração (CR), peso corporal (PC), ganho de peso (GP) e conversão alimentar (CA) de frangos de corte submetidos a restrição de água nos períodos de 8 a 14, 1 a 21 e 22 a 42 dias de idade

Table 2 - Water intake (WI), feed intake (FI), body weight (BW), weight gain (WG) and feed conversion (CA) of broilers submitted to different levels of water restriction, from 8 to 14,1 to 21 and 22 to 42 days old

Nível de Consumo de água $(\mathrm{mL}) *$ Consumo de ração $(\mathrm{g}) *$ Peso corporal $(\mathrm{g}) *$ Ganho de peso $(\mathrm{g}) *$ Conversão alimentar $(\mathrm{g} / \mathrm{g})$ $\begin{array}{lllll}\text { restrição (\%) Water intake } & \text { Feed intake } & \text { Body weight } & \text { Feight gain conversion }\end{array}$ Restriction level

\begin{tabular}{lrr}
\hline & & \\
0 & $916 \mathrm{~b}$ & 365 \\
10 & $1032 \mathrm{a}$ & 351 \\
20 & $1095 \mathrm{a}$ & 340 \\
30 & $1080 \mathrm{a}$ & 338 \\
40 & $1096 \mathrm{a}$ & 332 \\
$\mathrm{CV} \%$ & 8,9 & 6,2 \\
$\mathrm{P}$ & 0,012 & 0,35
\end{tabular}

8 a 14 dias (8 to 14 days)

$\begin{array}{lr}0 & 2872 \\ 10 & 3001 \\ 20 & 3001 \\ 30 & 2902 \\ 40 & 2914 \\ \mathrm{CV} & 7,9 \\ \mathrm{P} & 0,81\end{array}$

$1129 \mathrm{a}$

$1117 \mathrm{a}$

$1081 \mathrm{ab}$

$1066 \mathrm{ab}$

$1014 \mathrm{~b}$

4,8

0,006

$444 \mathrm{a}$
$442 \mathrm{a}$
$408 \mathrm{~b}$
$421 \mathrm{~b}$
$406 \mathrm{~b}$
4,9
0,007

$260 \mathrm{~b}$

$282 \mathrm{a}$

$268 \mathrm{ab}$

$286 a$

$281 \mathrm{a}$

4,3

0,003

1 a 21 dias (1 to 21 days)

$\begin{array}{rrr}850 & 807 & 1,39 \\ 848 & 805 & 1,39 \\ 811 & 768 & 1,41 \\ 827 & 784 & 1,36 \\ 808 & 765 & 1,32 \\ 4,2 & 4,4 & 4,7 \\ 0,12 & 0,12 & 0,18\end{array}$

22 a 42 dias (22 to 42 days)

2987

2939

3015

2927

2988

4,5

0,77

$\mathrm{CAg}=962,5+4,07 \mathrm{x} ; \mathrm{R}^{2}=0,37 ; \mathrm{P}<0,0001$

$\begin{array}{rr}1598 & 2448 \\ 1651 & 2500 \\ 1689 & 2500 \\ 1666 & 2493 \\ 1727 & 2535 \\ 7,1 & 4,8 \\ 0,44 & 0,81\end{array}$

1,87

1,79

1,78

1,75

1,73

6,4

0,30

$1,34 \mathrm{a}$

, $27 \mathrm{ab}$

$1,18 \mathrm{~b}$

$1,18 \mathrm{~b}$

5,4 001

*Médias seguidas de letras diferentes na mesma coluna diferem $(P<0,05)$ pelo teste Tukey.

* Means followed by different letter within a column are different $(P<0.05)$ by Tukey test.

Regressões lineares $(P<0,04)$ (Linear regression, $P<0.04)$. 
rendimento de carcaça e dos cortes (Tabela 3). Segundo Doyle \& Leeson (1997), os efeitos do crescimento compensatório na composição da carcaça ainda não estão bem definidos. Resultados de pesquisas sugerem que a restrição no início da vida das aves reduz o teor de gordura da carcaça na idade de abate uma vez que a restrição reduz a hiperplasia dos adipócitos (Plavnik \& Hurwitz, 1985). Entretanto, este fato não foi confirmado neste experimento. Os elevados coeficientes de variação para gordura abdominal dificultam qualquer conclusão com significado biológico sobre esta variável.

O coeficiente de variação do peso corporal foi medido até o período de 21 dias para verificar a uniformidade dos lotes. Esperava-se encontrar menor uniformidade com o aumento do nível de restrição, como se observa nos animais a campo, em virtude da dominância de frangos maiores na posse pela água. Os resultados, porém, não indicaram aumento de desuniformidade dos lotes e apresentaram coeficientes de variação bastante semelhantes entre os níveis de restrição. Provavelmente as condições experimentais apresentaram poucos desafios a ponto de causar disparidade de peso entre as aves, pois a área de bebedouro recomendada para frangos de corte é de $2,5 \mathrm{~cm}$ lineares de bebedouro calha por ave (Avisite, 2005) e neste experimento a área foi de 3,5 cm por ave, ou seja, $40 \%$ superior ao recomendado, fato que pode ter colaborado para a maior uniformidade dos lotes.

Observou-se aumento linear do percentual de hematócrito (Tabela 4) conforme aumentou a severidade da restrição aos 3, 7 e aos 28 dias de idade. As equações de regressão também indicaram que aos 7 dias de idade a relação entre hematócrito e desidratação foi maior que aos 3 dias de idade, o que gerou coeficiente de determinação maior $(0,42)$. Considerando a facilidade de mensuração, a análise de hematócrito pode ser uma ferramenta para determinar a campo a desidratação de frangos. Logo após o término da restrição de água, a análise de regressão indicou inversão nos resultados, provavelmente em decorrência do elevado consumo de água neste período pelas aves submetidas a restrição no período anterior. Os dados deste expe-

Tabela 3 - Rendimento de carcaça e dos cortes da carcaça aos 42 dias de idade de frangos submetidos a diferentes níveis de restrição de água

Table 3 - Yield of carcass and cuts of carcass at 42 days of age of broilers submitted to different levels of water restriction

\begin{tabular}{|c|c|c|c|c|c|c|}
\hline \multirow[t]{2}{*}{$\begin{array}{l}\text { Nível de restrição (\%) } \\
\text { Restriction level }\end{array}$} & \multicolumn{6}{|c|}{$\begin{array}{c}\text { Corte* } \\
\text { Cut }\end{array}$} \\
\hline & $\begin{array}{l}\text { Carcaça } \\
\text { Carcass }\end{array}$ & $\begin{array}{l}\text { Peito } \\
\text { Breast }\end{array}$ & $\begin{array}{c}\text { Dorso } \\
\text { Back }\end{array}$ & $\begin{array}{l}\text { Coxa } \\
\text { Thighs }\end{array}$ & $\begin{array}{c}\text { Perna } \\
\text { Leg }\end{array}$ & $\begin{array}{c}\text { Gord** } \\
\text { Abdominal fat }\end{array}$ \\
\hline 0 & 77,0 & 37,3 & 13,1 & 13,0 & 20,0 & $1,9 \mathrm{ab}$ \\
\hline 10 & 78,6 & 36,7 & 13,0 & 13,0 & 19,5 & $2,2 \mathrm{a}$ \\
\hline 20 & 78,0 & 36,8 & 13,0 & 13,2 & 20,2 & $2,2 \mathrm{a}$ \\
\hline 30 & 81,4 & 37,5 & 13,3 & 13,4 & 19,6 & $1,8 \mathrm{~b}$ \\
\hline 40 & 78,4 & 37,5 & 13,3 & 13,3 & 20,0 & $2,2 \mathrm{ab}$ \\
\hline $\mathrm{CV} \%$ & 10,3 & 4,8 & 10,8 & 6,2 & 8,0 & 21,2 \\
\hline Valor de $\mathrm{P}$ ( $P$ value $)$ & 0,39 & 0,40 & 0,84 & 0,32 & 0,56 & 0,01 \\
\hline
\end{tabular}

** Médias seguidas de letras diferentes na mesma coluna diferem $(P<0,01)$ pelo teste de Tukey.

** Means followed by a different letter within a column are different $(P<0.01)$ by Tukey test.

Tabela 4 - Percentual de hematócrito sangüíneo de frangos de corte submetidos a diferentes níveis de restrição de água Table 4 - Blood hematocrit percentage, of broilers submitted to different levels of water restriction

\begin{tabular}{|c|c|c|c|c|c|c|}
\hline \multirow[t]{2}{*}{$\begin{array}{l}\text { Nível de restrição (\% } \\
\text { Restriction level }\end{array}$} & \multicolumn{6}{|c|}{$\begin{array}{l}\text { Dia do experimento } \\
\text { Experimental day }\end{array}$} \\
\hline & $3 * *$ & $7 * *$ & $14 * *$ & 21 & 28 & 35 \\
\hline 0 & $24 b *$ & $29 b *$ & 37 & 31 & 30 & 35 \\
\hline 10 & $26 a b$ & $31 \mathrm{ab}$ & 35 & 34 & 32 & 32 \\
\hline 20 & $26 \mathrm{ab}$ & $30 \mathrm{~b}$ & 37 & 32 & 34 & 35 \\
\hline 30 & $26 \mathrm{ab}$ & $34 \mathrm{ab}$ & 34 & 34 & 34 & 35 \\
\hline CV\% & 8,3 & 9,8 & 7,9 & 6,2 & 8,3 & 12,4 \\
\hline \multirow[t]{2}{*}{ Valor de $\mathrm{P}$ ( $P$ value $)$} & 0,05 & 0,002 & 0,12 & 0,10 & 0,07 & 0,67 \\
\hline & \multicolumn{6}{|c|}{$\begin{array}{l}\text { Ht } 3 \text { dias }=24,5+0,08 x ; R^{2}=0,26 ; \mathrm{P}<0,004 \\
\text { Ht } 7 \text { dias }=28,4+0,18 \mathrm{x} ; \mathrm{R}^{2}=0,42 ; \mathrm{P}<0,0001 \\
\text { Ht } 14 \text { dias }=37,1-0,08 \mathrm{x} ; \mathrm{R}^{2}=0,17 ; \mathrm{P}<0,02\end{array}$} \\
\hline
\end{tabular}

\footnotetext{
* Médias seguidas de letras diferentes na mesma coluna diferem $(P<0,05)$ pelo teste Tukey.

* Means followed by a different letter in the columm are different $(P<0.05)$ by Tukey test.

Regressões lineares $(P<0,02)$ (Linear regression, $P<0.02)$.
} 
período de restrição. Esses autores sugeriram que neste período o consumo de alimento é maior em relação ao peso corporal, fato explicado pela maior capacidade digestiva do animal durante a fase de realimentação, atribuída à maior ingestão e ao maior espaço relativo disponível no trato digestório (Hornick et al., 2000; Lawrence \& Fowler, 2002).

Os níveis de restrição nos primeiros 21 dias (Tabela 6) influenciaram linear e negativamente os consumos de ração e de água, o ganho de peso e o peso corporal aos 21 dias. Apesar da recuperação verificada na semana de 8 a 14 dias, aos 21 dias ainda não foi possível a total compensação das perdas impostas na primeira semana. Sabe-se que o tempo de realimentação é importante para as aves recuperarem seus pesos (Lawrence \& Fowler, 2002). Todas as variáveis tiveram comportamento negativamente associado à severidade da restrição de ração. No entanto, a conversão alimentar não foi influenciada pelas restrições alimentares. Plavnik \& Hurwitz (1988) constataram que frangos sob restrição alimentar na fase inicial de vida apresentaram melhor conversão alimentar aos 45 dias. Acar et al. (2001) não registraram diferenças na conversão alimentar no período de 21 a 49 dias de idade em aves submetidas à restrição de $75 \%$ da EM durante 4 a 11 dias de idade.

Do mesmo modo que no experimento 1 , o coeficiente de variação do peso corporal medido até o período de 21 dias para verificar uniformidade não indicou aumento de desuniformidade dentro das repetições.

No período de 22 a 42 dias, as diferenças decorrentes da restrição de ração aplicada na primeira semana de vida desapareceram, comprovando total recuperação das aves (Tabela 6). Dados semelhantes foram observados experimentalmente por Vargas Jr. et al. (1995). No entanto, no estudo de Acar et al. (2001), a restrição de 25\% da EM no período de 4 a 11 dias de idade resultou em frangos $4 \%$ menores ao abate. Sugeta et al. (2002), aplicando restrições quantitativas de 30 ou $70 \%$ da ração, também verificaram

Tabela 6 - Consumo de ração (CR), consumo de água (CAg), peso corporal (PC), ganho de peso (GP) e conversão alimentar (CA) de frangos de corte submetidos a diferentes níveis de restrição alimentar nos períodos de 8 a 14,1 a 21 e 22 a 42 dias

Table 6 - Feed intake (CR), water intake (CAg), body weight (GP), weight gain (GP), feed conversion(CA), of broilers submitted to different levels of feed restriction from 8 at 14,1 to 21 and 22 to 42 days

Nível de Consumo de ração $(\mathrm{g}) *$ Consumo de água $(\mathrm{mL})^{*}$ Peso corporal $(\mathrm{g}) * *$ Ganho de peso $(\mathrm{g}) * *$ Conversão alimentar $(\mathrm{g} / \mathrm{g})$ restrição (\%) Feed intake Water intake Body weight $\quad$ Weight gain $\quad$ Feed conversion Restriction level

$\begin{array}{lrr}0 & 370 & 849 \\ 10 & 387 & 871 \\ 20 & 362 & 818 \\ 30 & 366 & 833 \\ 40 & 357 & 833 \\ \mathrm{CV} \% & 5,6 & 4,9 \\ \text { Valor de P (P value) } & 0,16 & 0,25\end{array}$

8 a 14 dias (8 to 14 days)

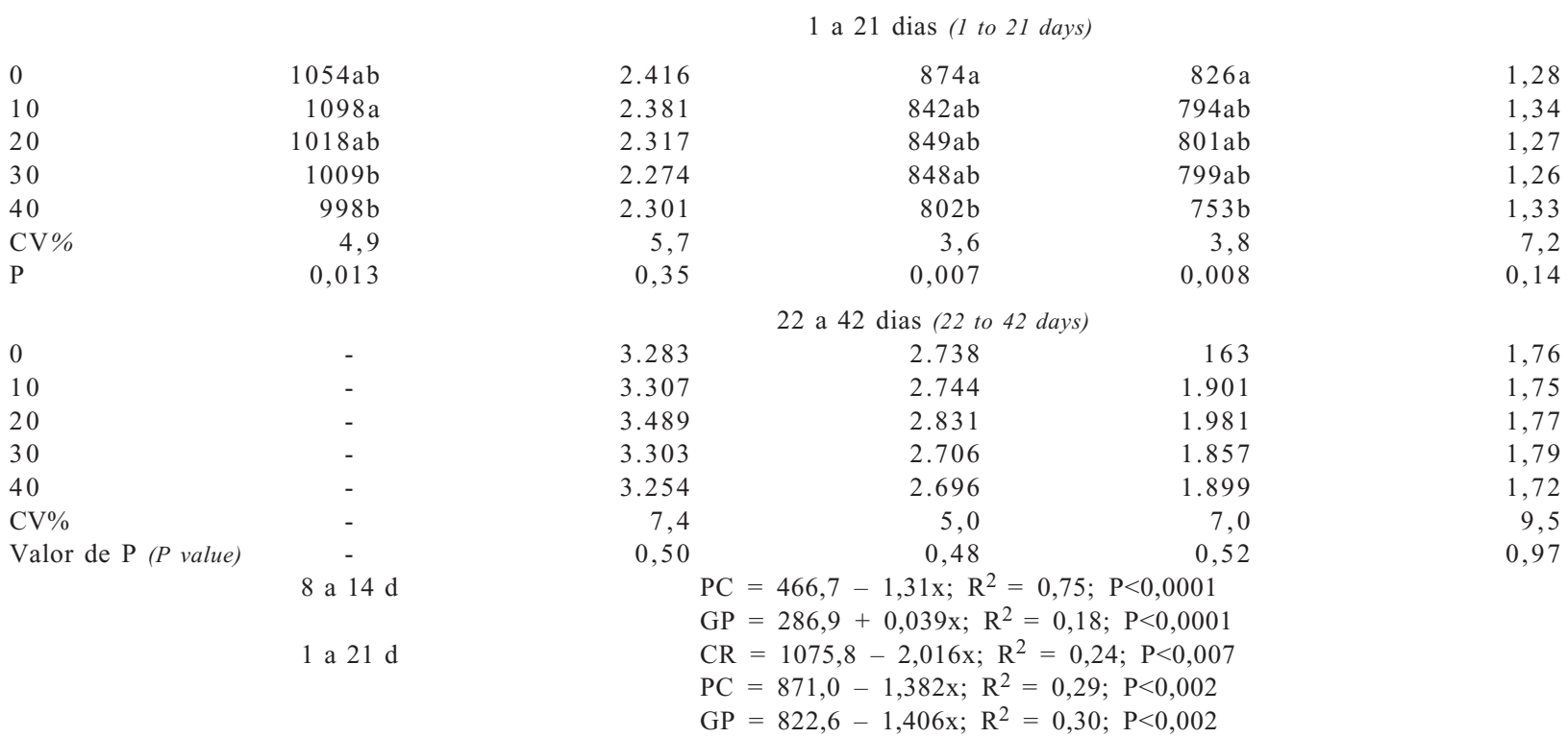

* Médias seguidas de letras diferentes na mesma coluna diferem $(P<0,05)$ pelo teste Tukey.

* Means followed by a different letter withina column are different $(\mathrm{P}<0.05)$ by Tukey test.

Regressões lineares $(P<0,002)$ (Linear regression, $P<0.002)$. 
piora no peso e no rendimento de carcaça das aves sob restrição de $70 \%$, sem diferença significativa na conversão alimentar.

A restrição de ração não influenciou os rendimentos de carcaça, de peito e de coxa (Tabela 7). Por outro lado, o rendimento de perna aumentou com a restrição de ração, ao contrário do rendimento de asa, embora com $\mathrm{R}^{2}$ muito pequenos. O percentual de gordura na carcaça não foi alterado pela restrição de ração. Yu \& Robinson (1992) relataram que, em alguns trabalhos, animais submetidos a restrição alimentar apresentaram menor teor de gordura abdominal. Deaton (1995), com restrição alimentar de 40\% para frangos dos 7 aos 14 dias de idade, observou redução de gordura abdominal nos animais sob restrição quando comparados aos animais do tratamento controle. No entanto, os autores reproduziram este experimento mais duas vezes e não observaram esse efeito novamente. Plavnik \& Hurwitz (1985) aplicaram restrição alimentar durante seis dias na primeira semana de vida dos frangos e observaram mesmo peso à idade de abate e porcentagem de gordura abdominal significativamente menor. Summer et al. (1990) não observaram redução da porcentagem de gordura abdominal ao aplicarem restrição alimentar no período dos 7 aos 14 dias de idade (restrição de 25 ou 50\%). De acordo com Doyle \& Leeson (1997), a restrição de ração atrasa a hiperplasia dos adipócitos, mas não impede sua hipertrofia, podendo resultar em aumento da gordura corporal.

A gordura abdominal não parece ser a mais recomendada como critério de verificação da deposição de gordura nas aves, visto que variações decorrentes da dificuldade de sua retirada da carcaça podem influenciar os resultados. Segundo Bazis et al. (1996), a gordura corporal seria a mais recomendada para esta comparação, uma vez que as aves apresentam quantidade significativa de deposição subcutânea de gordura. Sugeta et al. (2002), avaliando o total de gordura corpórea, observaram que os frangos sob restri-

Tabela 7 - Rendimento (\%) dos cortes da carcaça de frangos de corte submetidos a diferentes níveis de restrição alimentar aos 42 dias de idade

Table 7 - Carcass and cuts yield (\%) of broilers submitted to different levelss of feed restriction at 42 days of age

\begin{tabular}{|c|c|c|c|c|c|c|c|}
\hline \multirow[t]{2}{*}{$\begin{array}{l}\text { Nível de restrição (\%) } \\
\text { Restriction level }\end{array}$} & \multicolumn{7}{|c|}{$\begin{array}{c}\text { Corte* } \\
\text { Cut }\end{array}$} \\
\hline & $\begin{array}{l}\text { Carcaça } \\
\text { Carcass }\end{array}$ & $\begin{array}{l}\text { Peito } \\
\text { Breast }\end{array}$ & $\begin{array}{l}\text { Dorso } \\
\text { Back }\end{array}$ & $\begin{array}{l}\text { Coxa } \\
\text { Thighs }\end{array}$ & $\begin{array}{c}\text { Perna } \\
\text { Leg }\end{array}$ & $\begin{array}{l}\text { Asa } \\
\text { Wing }\end{array}$ & $\begin{array}{c}\text { Gord** } \\
\text { Abdominal fat }\end{array}$ \\
\hline 0 & 73,8 & 37,7 & $12,6 a b$ & 13,8 & 21,0 & $10,6 a b$ & 1,8 \\
\hline 10 & 72,8 & 38,3 & $12,5 \mathrm{ab}$ & 13,8 & 21,1 & $10,7 \mathrm{a}$ & 1,8 \\
\hline 20 & 73,8 & 39,3 & $12,2 b$ & 13,6 & 21,6 & $10,4 a b$ & 1,7 \\
\hline 30 & 72,1 & 39,4 & $13,0 \mathrm{a}$ & 14,9 & 21,6 & $10,5 \mathrm{ab}$ & 1,7 \\
\hline 40 & 73,8 & 38,8 & $12,4 \mathrm{ab}$ & 13,9 & 21,7 & $10,3 b$ & 1,8 \\
\hline CV\% & 4,4 & 11,8 & 6,8 & 5,5 & 6,1 & 4,9 & 30,7 \\
\hline Valor de $\mathrm{P}$ ( $P$ value $)$ & 0,26 & 0,71 & $\begin{array}{r}0,028 \\
\text { Perna }=21 \\
\text { Asa }=10,7\end{array}$ & $\begin{array}{l}0,52 \\
\mathrm{x} ; \mathrm{R}^{2}= \\
\mathrm{R}^{2}=0\end{array}$ & $\begin{array}{c}0,23 \\
\mathrm{P}<0,0332 \\
0,0124\end{array}$ & 0,025 & 0,83 \\
\hline
\end{tabular}

* Médias seguidas de letras diferentes na mesma coluna diferem $(P<0,05)$ pelo teste Tukey.

* Means followed by a different letter within a column are different $(\mathrm{P}<0.05)$ by Tukey test.

Tabela 8 - Percentual de hematócrito sanguíneo de frangos de corte submetidos a diferentes níveis de restrição alimentar Table 8 - Blood hematocrit percentage of broilers submitted to different levels of feed restriction

\begin{tabular}{|c|c|c|c|c|c|c|}
\hline \multirow[t]{2}{*}{$\begin{array}{l}\text { Nível de restrição (\%) } \\
\text { Restriction level }\end{array}$} & \multicolumn{6}{|c|}{$\begin{array}{l}\text { Dia do experimento } \\
\text { Experimental day }\end{array}$} \\
\hline & $3 * *$ & 7 & 14 & 21 & 28 & 35 \\
\hline 0 & $28 \mathrm{bc} *$ & 33 & 35 & 32 & 30 & 33 \\
\hline 10 & $26 \mathrm{c}$ & 32 & 33 & 31 & 29 & 32 \\
\hline 20 & $33 \mathrm{ab}$ & 34 & 34 & 33 & 31 & 34 \\
\hline 30 & $35 \mathrm{a}$ & 35 & 33 & 36 & 29 & 35 \\
\hline 40 & $34 \mathrm{ab}$ & 34 & 33 & 32 & 28 & 36 \\
\hline CV\% & 11,2 & 8,5 & 8,1 & 11,0 & 6,9 & 9,6 \\
\hline \multirow[t]{2}{*}{ Valor de $\mathrm{P}$ (P value } & 0,001 & 0,69 & 0,45 & 0,20 & 0,17 & 0,31 \\
\hline & \multicolumn{6}{|c|}{ Hematócrito $3=27,7+0,19 x ; R^{2}=0,33 ; P<<0,0007$} \\
\hline
\end{tabular}

* Médias seguidas de letras diferentes na mesma coluna diferem $(P<0,05)$ pelo teste Tukey.

${ }^{*}$ Means followed by a different letter within a column are different $(P<0.05)$ by Tukey test. Regressões lineares $(P<$ ? $)$ Linear regression $(P<$ ? $)$ 
ção alimentar de $70 \%$ tiveram menor teor de gordura total em comparação aos frangos sob $30 \%$ de restrição ou alimentados à vontade.

Aos 3 dias de idade, observou-se efeito da restrição alimentar no aumento do percentual de hematócrito (Tabela 8). No entanto, esse resultado não persistiu estaticamente até os 7 dias de idade, período no qual os frangos também ingeriram menor quantidade de água. Verificando o consumo de água em relação ao de ração, constatou-se que os animais sob restrição de 30 ou $40 \%$ consumiram proporcionalmente mais água, o que justifica essa ausência de diferença no valor de hematócrito.

Comparando os pesos relativos dos órgãos analisados (peso do órgão/peso corporal), somente o peso da moela aos 7 dias de idade aumentou com a restrição de ração $\left(\hat{Y}=4,97+0,107 x ;\right.$ Prob $\left.=0,0001 ; R^{2}=0,37\right)$. Os pesos dos demais órgãos não diferiram, embora tenham diminuído proporcionalmente com a diminuição no peso corporal na análise dos pesos absolutos.

Na Figura 1 é possível observar a evolução das vilosidades dos 7 aos 21 dias experimentais de acordo com os níveis de restrição de água (experimento 1). As fotos indicam diferença visual entre as vilosidades no intestino das aves sem restrição controle e daquelas sob $40 \%$ de restrição de água. Nas aves criadas com água à vontade, as vilosidades apresentaram-se com aspecto mais liso e arredondado, enquanto o aumento da restrição parece ter aumentado as alterações na superfície, resultando em vilosidades mais achatadas e enrugadas. O dano causado pela restrição até os 7 dias de idade parece permanecer até os 21 dias, sem recuperação da integridade da mucosa. Essa observação confirma os relatos de Maiorka et al. (2001), que verificaram efeito prejudicial da restrição de água e de ração sobre a mucosa intestinal em frangos de corte e corroboram os dados de desempenho, que comprovaram que até os 21 dias as aves sob restrição não haviam se recuperado totalmente.

As fotos dos experimentos 1 e 2 foram similares, exceto pelo fato de que não há diferenças visuais entre as vilosidades do intestino das aves criadas sem restrição e o daquelas sob $40 \%$ de restrição alimentar. Portanto, neste estudo, são apresentadas somente as fotos dos obtidas com os dois níveis extremos de restrição (Figura 2).
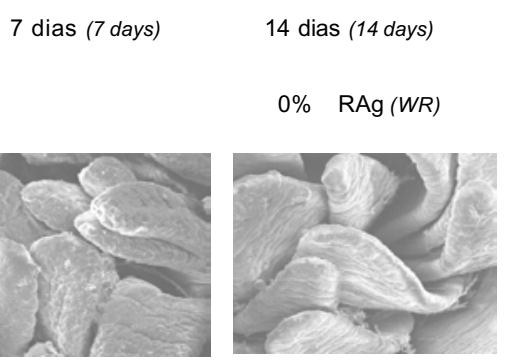

21 dias (21 days)

$10 \% \quad \operatorname{RAg}(W R)$
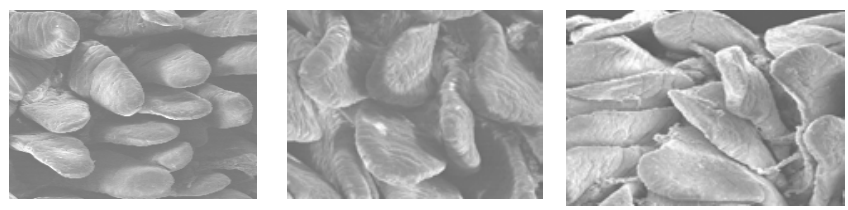

$20 \% \operatorname{RAg}(W R)$
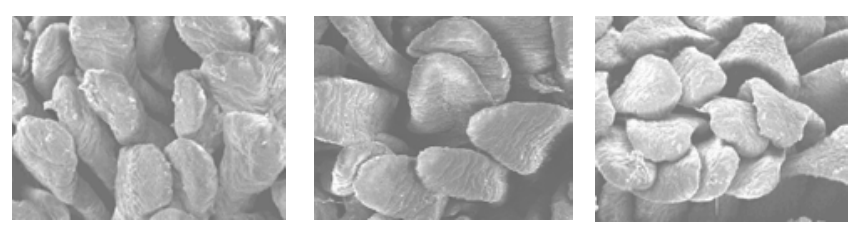

$30 \% \quad \operatorname{RAg}(W R)$
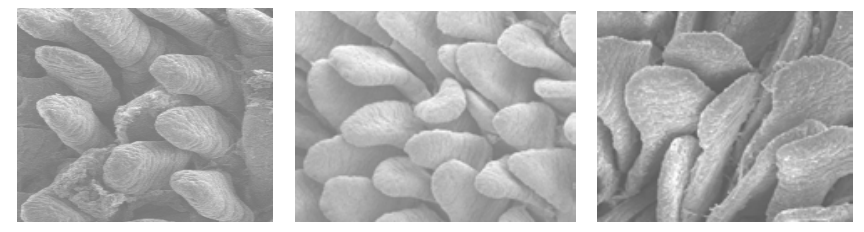

$40 \% \quad \operatorname{RAg}(W R)$
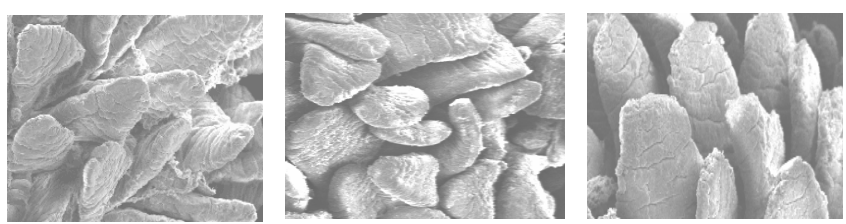

Figura 1 - Evolução das vilosidades intestinais de frangos de corte submetidos à restrição de água (RAg) na primeira semana de vida (experimento 1).

Figure 1 - Villi evolution of broilers submitted to water restriction (RAg) in the first week of life (experiment 1) 
$0 \% \quad \operatorname{RR}(W R)$
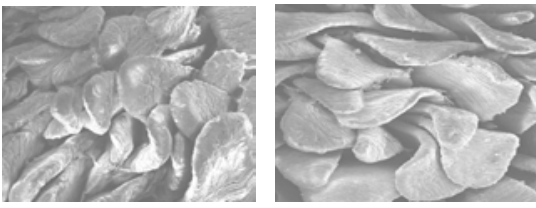

$40 \% \quad \operatorname{RR}(W R)$
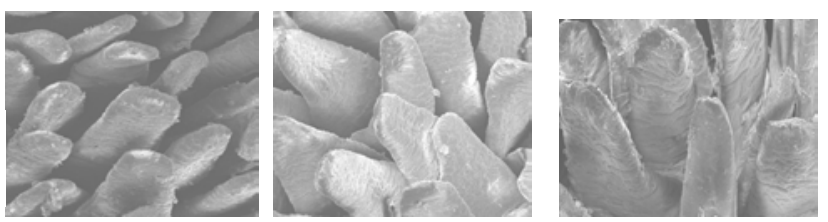

Figura 2 - Evolução das vilosidades intestinais de frangos de corte submetidos a restrição de ração (RR) na primeira semana de vida (experimento 2).

Figure 2 - Villi evolution of broilers submitted to feed restriction $(R R)$ in the first week of life (experiment 2).

\section{Conclusões}

Os consumos de água e ração são altamente correlacionados em frangos de corte, uma vez que a restrição de um afeta o consumo do outro componente, embora a restrição de água tenha maior influência na redução do consumo alimentar. Tanto a restrição de água quanto a de ração na primeira semana de vida afetam o desempenho e o peso de órgãos, porém, a proporcionalidade entre o peso dos órgãos e o peso corporal se mantém, com exceção da moela, que aumenta proporcionalmente ao peso. No entanto, as aves submetidas à qualquer forma de restrição, em condições experimentais, tiveram capacidade de recuperação do desempenho aos 42 dias e apresentaram crescimento compensatório, sobretudo na semana subseqüente à restrição. Os dados de hematócrito são um parâmetro adequado de avaliação da desidratação em frangos de corte.

\section{Literatura Citada}

ACAR, N.; SIZEMORE, F.Z.; LEACH, G.R. et al. Growth of broiler chickens in response to feed restriction regimes to reduce ascites. Poultry Science, v.74, p.833-843, 2001.

BAZIZ, H.A.; GERAERT, P.A.; PADILHA, J.C.F. et al. Chronic heat exposure enhances fatdeposition and modifies muscle and fat partition in broiler carcasses. Poultry Science, v.75, n.4, p.505-513, 1996 .

BROOKS, P.H. Water - Forgotten nutrient and novel delivery system. In: ALLTECH'S ANNUAL SYMPOSIUM, 10., 1994, Gaiswill. Proceedings... Nottingham: Nottingham University Press, 1994. p.211-234.

COUNOTTE, G. Avicultura profesional: Conocer la calidad del água de bebida. Doetinchem: Reed Business Information, 2003. p. 20-22.
DEATON, J.W. The effect of early feed restriction on broiler performance. Poultry Science, v.74, p.1280-1286, 1995.

DIBNER, J.J.; KITCHELL, M.L.; ATWELL, C.A. et al. The effect of dietary ingredients and age on the microscopic structure ofthe gastrointestinal tract in poultry. Journal of Applied Poultry Research, v.5, n.1, p.70-77, 1996.

DOYLE, F.; LEESON, S. Compensatory growth farms animals. II. Methods of implementation and animal performance. Guelph, Ontario: Department of Animal and Poultry Science, University Guelph, 1997. p.85-103.

FONTANA, E.A.; WEAVER, W.D.; WATKINS, B.A. et al. Effect of early feed restriction on growth, feed conversion, and mortality in broiler chickens. Poultry Science, v.71, p.1296-1305, 1993.

HORNICK, J.L.; EENAEME, C.; GÉRARD, O. et al. Mechanisms of reduced and compensatory growth. Domestic Animal Endocrinology, v.19, n.2, p.121-132, 2000.

VARGAS JR., J.G.V.; ALBINO, L.F.T.; ROSTAGNO, H.S. et al. Desempenho de frango de corte submetidos a restrição alimentar. Revista Brasileira de Zootecnia, v.24, n.5, p.8082, 1995 .

LARBIER, M.; LECLERCQ, B. Nutrition and feeding of poultry: Intake of food and water. Nottingham: Nottingham University Press, 1994. p.7-14.

LAWRENCE, T.L.J.; FOWLER, V.R. Growth of farm animals. 2.ed. Aberdeen: CAB International, 2002. 368p.

LLOYD, L.E.; McDONALD, B.E.; CRAMPTON, E.W. Fundamentals of nutrition: water and its metabolism. San Francisco: W.H. Freeman and Company, 1978. p.22-35.

MACARI, M. Metabolismo hídrico da poedeira comercial. In: SIMPÓSIO TÉCNICO DE PRODUÇÃO DE OVOS, 5., 1995, Jaboticabal. Resumos... Jaboticabal: APA, 1995. p.109-131.

MAIORKA, A.; SANTIN, E.; DALHKE, F. et al. Effect of feed and/or water withdrawal on intestinal mucosa development in broiler chicks after hatching. Poultry Science, v.80, n.1, p.393, 2001. (Abstracts).

NILIPOUR, A.H.; BUTCHER, G.D. Water: the cheap, plentiful and taken for granted nutrient. World Poultry, v.14, n.1, p.26$27,1998$.

NIR, I.; LEVANON, M. Effect of posthatch holding time on performance and on residual yolk and liver composition. Poultry Science, v.72, n.12, p.1994-1997, 1993.

NITSAN, Z.; BEM-AURAHAM, G.; ZOREF, Z. et al. Growth and development of the digestive organs and some enzymes in broiler chicks after hatching. British Poultry Science, v.32, p.515523, 1991.

NOY, Y.; SKLAN, D. Different types of early feeding and performance in chicks and poults. Journal of Applied Poultry Research, v.8, n.1, p.16-24, 1999.

PLAVNIK, I.; HURWITZ, S. The performance of broiler chicks during and following a severe feed restriction at an early age. Poultry Science, v.64, p.348-355, 1985.

PLAVNIK, I.; HURWITZ, Early feed restriction in chicks: Effect of age, duration, and sex. Poultry Science, v.67, p.384390,1988 .

ROSA, P.S.; ÁVILLA, V.S.; JAENISCH, F.R.F. Restrição alimentar em frangos de corte: como explorar suas potencialidades. Concórdia: Embrapa Suínos e Aves, 2000. p.1-4 (Comunicado Técnico).

SUGETA, S.M.; GIACHETTO, P.F.; MALHEIROS, E.B. et al. Efeito da restrição alimentar quantitativa sobre o ganho compensatório e composição da carcaça de frangos. Pesquisa Agropecuária Brasileira, v.5, n.7, p.903-908, 2002.

SUSBILLA, J.P.; FRANKEL, T.L.; PARKINSON, G. et al. Weight of internal organs and carcase yield of early food restricted broilers. British Poultry Science, v.35, p.677$685,1994$.

STATISTICAL ANALYSIS SYSTEM - SAS. Painless Windows, a handbook for SAS users. 2.ed. Guelph: Jodie Gilmore, 2001. $61 \mathrm{p}$. 
ShlOSBERG, A.; BELLAICHE, M.; BERMAN, E. et al. A. Comparative effects of added sodium chloride, ammonium chloride, or potassium bicarbonate in the drinking water of broilers, and feed restriction, on the development of the ascites syndrome. Poultry Science, v.77, n.5, p.1287-1296, 1998.

VIOLA, T.; RIBEIRO, A.M.L.; PENZ JR., A.M. Compensatory water consumption of broilers submitted to water restriction from 1 to 21 days of age. Brazilian Journal of Poultry Science, v.7, n.4, p.243-246, 2005.
YU, M.W.; ROBINSON, F.E. The application of short-term feed restriction to broiler chicken production: a review. Journal of Applied Poultry Research, v.1, n.1, p.147-153, 1992.

Recebido: 1/9/2006 Aprovado: 18/4/2007 